INPLASY

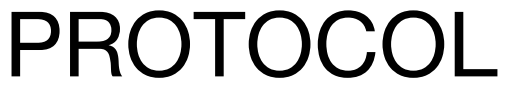

To cite: Guan et al.

Effectiveness comparisons of acupuncture treatments for Vascular dementia: A protocol for systematic review and network meta-analysis. Inplasy protocol 2020110088. doi: 10.37766/inplasy2020.11.0088

Received: 19 November 2020

Published: 20 November 2020

Corresponding author:

Kangfeng Wang

szywkf@163.com

Author Affiliation:

First College of Clinical Medicine, Shandong University of Traditional Chinese Medicine

Support: Serial number: 81303028.

Review Stage at time of this submission: Piloting of the study selection process.

Conflicts of interest: The authors have no conflicts of interest to disclose.

\section{Effectiveness comparisons of acupuncture treatments for Vascular dementia: A protocol for systematic review and network meta-analysis}

Guan, X1; Zhang, L2; Wu, T3; Zhi, H4; Hou, H5; Bi, S6; Wang, K7.

Review question / Objective: The aim of this network metaanalysis is to evaluate the efficacy and safety of acupuncture treatments for Vascular dementia.

Condition being studied: Vascular dementia (VD) is an irreversible cognitive disorder closely related to age. Vascular dementia is the second leading cause of dementia after AD, accounting for at least $\mathbf{2 0 \%}$ of dementia cases. It is still an urgent problem to seek effective treatment methods.

Information sources: We will search the following sources regardless of date, language, or publication status: PubMed, Embase, Cochrane, Web of Science, China National Knowledge Infrastructure (CNKI), Wanfang Database, Chinese Biomedical Literature Database (CBM), Chinese Scientific Journals Database (VIP) database, and ongoing clinical trials registered on the International Clinical Registration Platform. The search date for the system is built to October 30,2020 . If information affecting NMA results is lacking in the literature, we will attempt to contact the original researchers for relevant information.

INPLASY registration number: This protocol was registered with the International Platform of Registered Systematic Review and Meta-Analysis Protocols (INPLASY) on 20 November 2020 and was last updated on 20 November 2020 (registration number INPLASY2020110088).

\section{INTRODUCTION}

Review question / Objective: The aim of this network meta-analysis is to evaluate the efficacy and safety of acupuncture treatments for Vascular dementia

Condition being studied: Vascular dementia (VD)is an irreversible cognitive disorder 
closely related to age. Vascular dementia is the second leading cause of dementia after $A D$, accounting for at least $20 \%$ of dementia cases. It is still an urgent problem to seek effective treatment methods.

\section{METHODS}

Participant or population: All patients diagnosed with VD will be included in the study, and the diagnostic criteria will be based on the definition of vascular dementia formulated by NINDS/Airen Clinical Criteria for the Diagnosis of Vascular Dementia, CCDVD. Age, sex, course of disease, severity of illness, nationality, race and duration will not be restricted.

Intervention: The intervention measures of the experimental group included common acupuncture, scalp acupuncture, acupuncture, ear, electro acupuncture acupoint catgut embedding, used alone or combination of any 2 methods, or in combination with western medicine which should be the same as that of the control group .Laser acupuncture, bee venom acupuncture, acupoint application, bleeding and acupressure will be excluded.

Comparator: The control group will use conventional western medicine or different acupuncture methods from the experimental group.

Study designs to be included: Randomized controlled clinical trials will be included.

Eligibility criteria: All randomized controlled clinical trials of acupuncture for vascular dementia published in Chinese or English will be included in the study.

Information sources: We will search the following sources regardless of date, language, or publication status: PubMed, Embase, Cochrane, Web of Science, China National Knowledge Infrastructure (CNKI), Wanfang Database, Chinese Biomedical Literature Database (CBM), Chinese Scientific Journals Database (VIP) database, and ongoing clinical trials registered on the International Clinical
Registration Platform. The search date for the system is built to October 30,2020 . If information affecting NMA results is lacking in the literature, we will attempt to contact the original researchers for relevant information.

Main outcome(s): The main outcomes are the vascular dementia Assessment Scale (VADAS-COG) and the Simple Mental State Examination (MMSE) scale, and adverse events(rash, itching, pain, etc.).

Quality assessment / Risk of bias analysis: Two researchers will independently assess the risk of bias in the included literature using the Cochrane Collaboration Risk of Bias Tool. Methods of random allocation generation, allocation concealment, participant blindness, outcome evaluator blindness, selective reporting, integrity of outcome data, and other sources of bias will be evaluated. Each field will be appropriately rated as high risk of bias and low risk of bias for uncertainty. The objection will be discussed by the two researchers to solve, if failed to agree on, will be settled by a third party to participate in.

Strategy of data synthesis: Two-by-two meta-analysis will be conducted using STATA15.0, and The odds ratio(OR) will be used as the effect indicator for the dichotomous variable, MD as the effect indicator for the continuous variable, and a $95 \% \mathrm{Cl}$ will be given for each effect index. Heterogeneity between studies was assessed by Cochrane $Q$ and $X 2$, and the size of heterogeneity was assessed by 12 . We will use Stata14.2 and WinBUGS1.4.3 software to perform bayesian network meta-analysis and merge the data in the random effects model.We will map the evidence network to represent comparisons between studies, with the thickness of the edges representing the number of comparisons made, and the size of the points representing the number of participants. Bayesian network algorithm USES Markov chain Monte Carlo method for reasoning. Inconsistencies between direct and indirect comparisons will be assessed by the node-splitting method. By 
comparing the deviation information standards of each model, consistent and inconsistent models, fixed-effect models and random-effect models were selected. The ranking of the effects of different acupuncture treatments will be presented by the surface under the cumulative ranking curve (SUCRA).

Subgroup analysis: If the heterogeneity is large, we will conduct a subgroup analysis of age, duration of treatment, course of VD and study quality to find the source of heterogeneity.

Sensibility analysis: We will conduct sensitivity analysis of outcome indicators and exclude references article by article. If the heterogeneity changes after excluding a certain reference, it indicates that the reference may be the source of heterogeneity. In addition, the reasons for heterogeneity can be explained by analyzing sample size, experimental design, outcome indicators, evaluation criteria, etc. If the heterogeneity remains unchanged after the exclusion of literature article by article, the results are relatively robust.

Country(ies) involved: China.

Keywords: acupuncture, Vascular dementia, network meta-analysis, protocol.

Contributions of each author:

Author 1 - Xiuju Guan.

Author 2 - Lijuan Zhang.

Author 3 - Tong Wu.

Author 4 - Hongwei Zhi.

Author 5 - Hanru Hou.

Author 6 - Shuyue Bi.

Author 7 - Kangfeng Wang. 